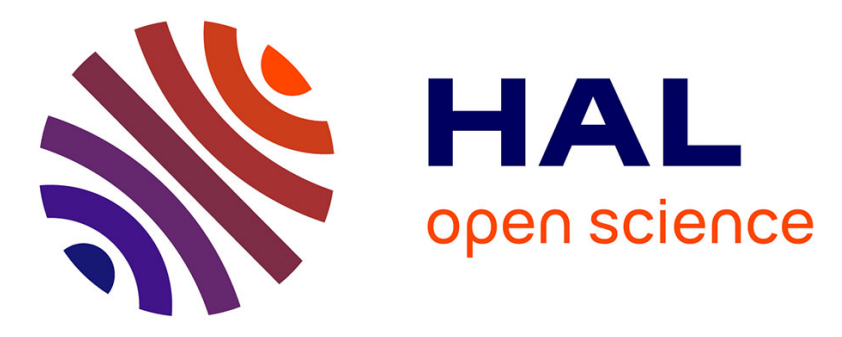

\title{
Premier signalement du Puceron Sarucallis kahawaluokalani (Kirkaldy, 1907) et de ses ennemis naturels en Guadeloupe (Hemiptera, Aphididae)
}

\author{
Jean Etienne, Bruno Michel, Cyril Grapin
}

\section{To cite this version:}

Jean Etienne, Bruno Michel, Cyril Grapin. Premier signalement du Puceron Sarucallis kahawaluokalani (Kirkaldy, 1907) et de ses ennemis naturels en Guadeloupe (Hemiptera, Aphididae). Bulletin de la Société Entomologique de France, 2018, 123 (4), pp.447-450. 10.32475/bsef_2049 . hal02624548

\author{
HAL Id: hal-02624548 \\ https://hal.inrae.fr/hal-02624548
}

Submitted on 26 May 2020

HAL is a multi-disciplinary open access archive for the deposit and dissemination of scientific research documents, whether they are published or not. The documents may come from teaching and research institutions in France or abroad, or from public or private research centers.
L'archive ouverte pluridisciplinaire HAL, est destinée au dépôt et à la diffusion de documents scientifiques de niveau recherche, publiés ou non, émanant des établissements d'enseignement et de recherche français ou étrangers, des laboratoires publics ou privés. 


\title{
Premier signalement du Puceron Sarucallis kahawaluokalani (Kirkaldy, 1907) et de ses ennemis naturels en Guadeloupe (Hemiptera, Aphididae)
}

\author{
Jean ÉtienNe ${ }^{1}$, Bruno Michel ${ }^{2} \&$ Cyril Grapin ${ }^{3}$ \\ ${ }^{1}$ INRA, Centre Antilles-Guyane, Domaine Duclos, F - 97170 Petit-Bourg, Guadeloupe < jean.etienne2@wanadoo.fr> \\ ${ }^{2}$ Cirad, UMR CBGP, 755 avenue du Campus Agropolis, CS30016, F - 34988 Montferrier-sur-Lez <bruno.michel@cirad.fr> \\ ${ }^{3}$ Desbonnes, F-97129 Lamentin, Guadeloupe <cglamgua@gmail.com>
}

(Accepté le 22.X.2018; publié le 3.XII.2018)

Résumé. - Le puceron Sarucallis kahawaluokalani (Kirkaldy) est signalé pour la première fois de Guadeloupe et plusieurs espèces de prédateurs qui lui sont associées sont mentionnées. Toutes les espèces identifiées sont illustrées.

Abstract. - First record of the crape myrtle aphid Sarucallis kahawaluokalani (Kirkaldy) and its natural enemies in Guadeloupe (Hemiptera, Aphididae). The aphid Sarucallis kahawaluokalani (Kirkaldy) is recorded for the first time from Guadeloupe and several predator species associated with it are mentioned. All the identified species are illustrated.

Keywords. - Crape myrtle aphid, new record, host-plant, predators, West Indies, Neotropical Region.

Un premier inventaire des Aphididae de la Guadeloupe avait permis de recenser 41 espèces (ÉTIENNE, 2005). Par la suite, deux autres espèces ont été signalées (ÉTIENNE \& CHAMPOISEAU, 2011) et la découverte récente de Sarucallis kahawaluokalani (Kirkaldy, 1907) porte à 44 le nombre des espèces de Pucerons inventoriées à ce jour en Guadeloupe.

Initialement décrit dans le genre Myzocallis par KIRKALDY (1907), Sarucallis kahawaluokalani (Kirkaldy, 1907) a été cité ou redécrit sous plusieurs noms : Agrioaphis kahawaluokalami [sic] (Kirkaldy, 1907), Callipterus kahawaluokalani (Kirkaldy, 1907), Monellia lagerstroemiae Takahashi, 1920, Neotherioaphis chhenafuli Behura \& Dash, 1975, Sarucallis lythrae Shinji, 1922, et Tinocallis kahawaluokalani (Kirkaldy, 1907) (FAVRET, 2018).

S. kahawaluokalani a été collecté pour la première fois en Guadeloupe par l'un d'entre nous (CG) le 8 novembre 2016, puis le 8 juin et le 3 novembre 2017 à Boubers (commune du Lamentin) sur Lagerstroemia indica L. (Lythraceae). Il s'agit d'une espèce essentiellement monophage qui est un ravageur important de cette plante ornementale. Ce Puceron a également été trouvé occasionnellement sur Lawsonia alba Lam. (Lythraceae) (BLACKMAN \& EASTOP, 1994), Eugenia uniflora L. (Myrtaceae) au Costa Rica (Zamora Mejías, 2009) et Punica granatum L. (Punicaceae) aux Philippines (Mizell \& Knox, 1993) sans provoquer de dégâts. Les deux dernières espèces sont présentes en Guadeloupe mais les prospections effectuées jusqu'à présent sur ces plantes n'ont pas permis d'y détecter la présence de Sarucallis kahawaluokalani.

Caractères diagnostiques. - S. kahawaluokalani est probablement le plus petit Puceron connu de Guadeloupe et ne peut pas être confondu avec d'autres espèces présentes dans l'île, en particulier celles susceptibles de se trouver sur Lagerstroemia indica. Divers auteurs (LECLANT \& Renoust, 1986 : Kondo \& Simbaqueba Cortés, 2014) en ont donné une description précise de sorte que nous n'indiquons ici que quelques caractéristiques spécifiques à cette espèce. Les ailés (fig. 1) ont la tête et le thorax jaune et noir avec des antennes de six segments. Les ailes sont tachées de noir notamment sur le bord antérieur et à l'apex, avec des nervures bordées de brun. L'abdomen jaune présente sur le dessus du deuxième segment un tubercule brun-noir 

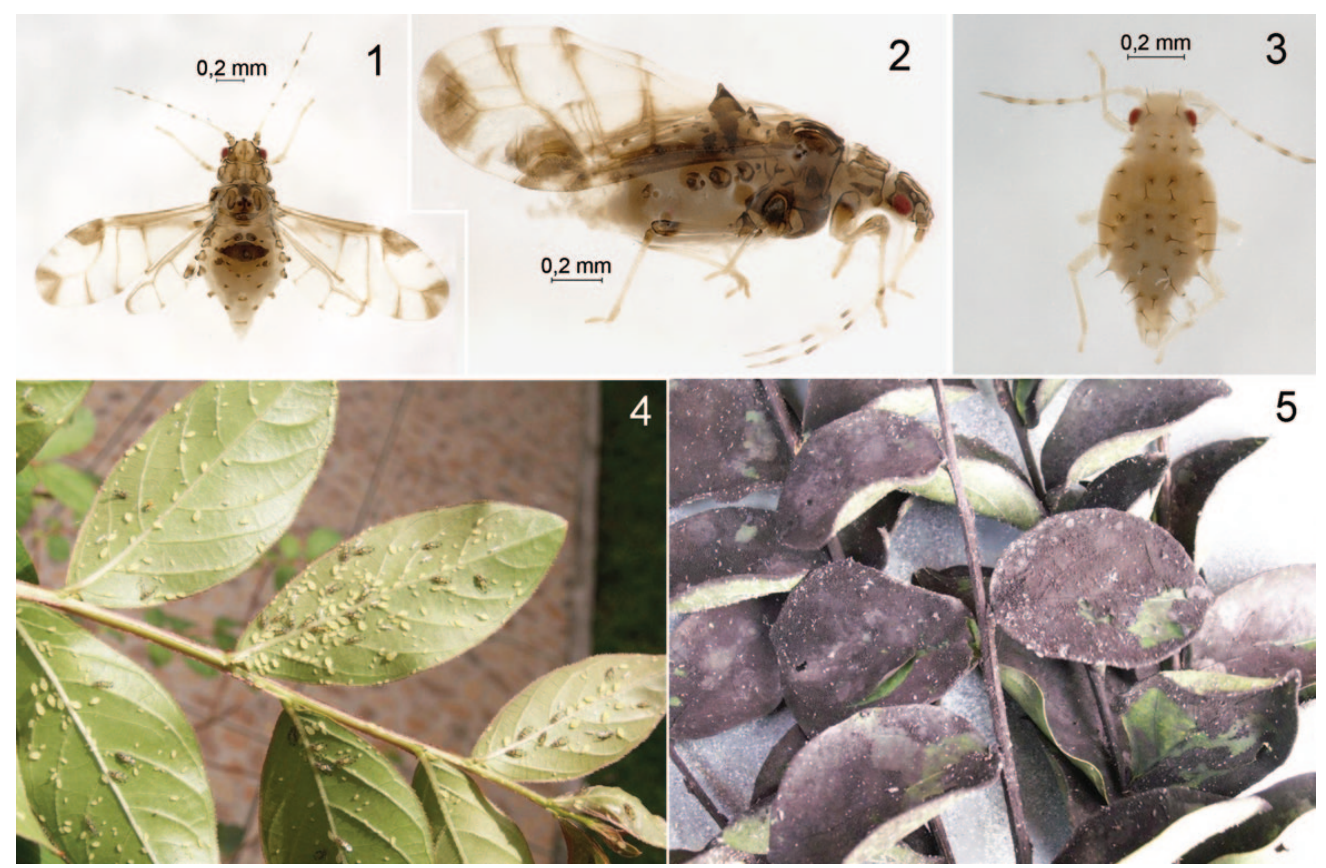

Fig. 1-5. - Sarucallis kahawaluokalani (Kirkaldy). - 1-2, habitus de l'adulte : 1, vue dorsale; 2, vue latérale. - 3, juvénile, vue dorsale. $\mathbf{- 4}$, colonies sur la face inférieure de feuilles de Lagerstroemia indica. - 5, fumagine sur feuilles de L. indica.

bifide caractéristique (fig. 2). Les cornicules noires sont très courtes. Les juvéniles (fig. 3) sont d'un jaune brillant avec de nombreuses soies noires érigées sur la partie dorsale de l'abdomen. C'est seulement lorsque l'espèce est bien installée sur le feuillage (fig. 4) qu'une abondante fumagine y apparaît et signale sa présence (fig. 5).

Ennemis naturels. - Aucun Hyménoptère parasitoïde de Sarucallis kahawaluokalani n'a été trouvé en Guadeloupe où les premières observations ont montré uniquement la présence de prédateurs généralistes. Il s'agit de trois Coléoptères Coccinellidae [Coelophora inaequalis (Fabricius, 1775) (fig. 6), Cycloneda delauneyi (Fleutiaux \& Sallé, 1889) (fig. 7), C. sanguinea (Linné, 1763) (fig. 8)], d'un Névroptère Chrysopidae [Ceraeochrysa cincta (Schneider, 1851) (fig. 9-10)] et de deux Diptères Syrphidae [Ocyptamus dimidiatus (Fabricius, 1781) (fig. 11), Allograpta limbata (Fabricius, 1805) (fig. 12)]. D'après Rojo et al. (2003) ces deux espèces de Syrphidae n'ont jamais été citées comme prédateurs de S. kahawaluokalani.

Toutes ces espèces ont été observées dans les colonies de $S$. kahawaluokalani à l'état larvaire, et également adulte pour les coccinelles, en train de s'alimenter sur des pucerons. La mise en élevage de larves de ces six espèces, collectées dans des colonies aphidiennes, a permis d'obtenir des adultes et de réaliser les identifications.

Un Hémiptère Anthocoridae, non déterminé, a été observé une seule fois dans une colonie de S. kahawaluokalani sans qu'on puisse affirmer qu'il est bien un prédateur de cette dernière espèce. Compte tenu du régime alimentaire des Anthocoridae, on peut penser qu'il peut également se nourrir aux dépens de ce puceron mais cette hypothèse reste à vérifier.

Ces premières collectes doivent être poursuivies afin de mieux connaître et inventorier les espèces prédatrices et peut-être trouver quelques parasitoïdes spécifiques ou non. Nos observations corroborent les travaux de Mizell et al. (2002) qui ont recherché sans succès d'éventuels 

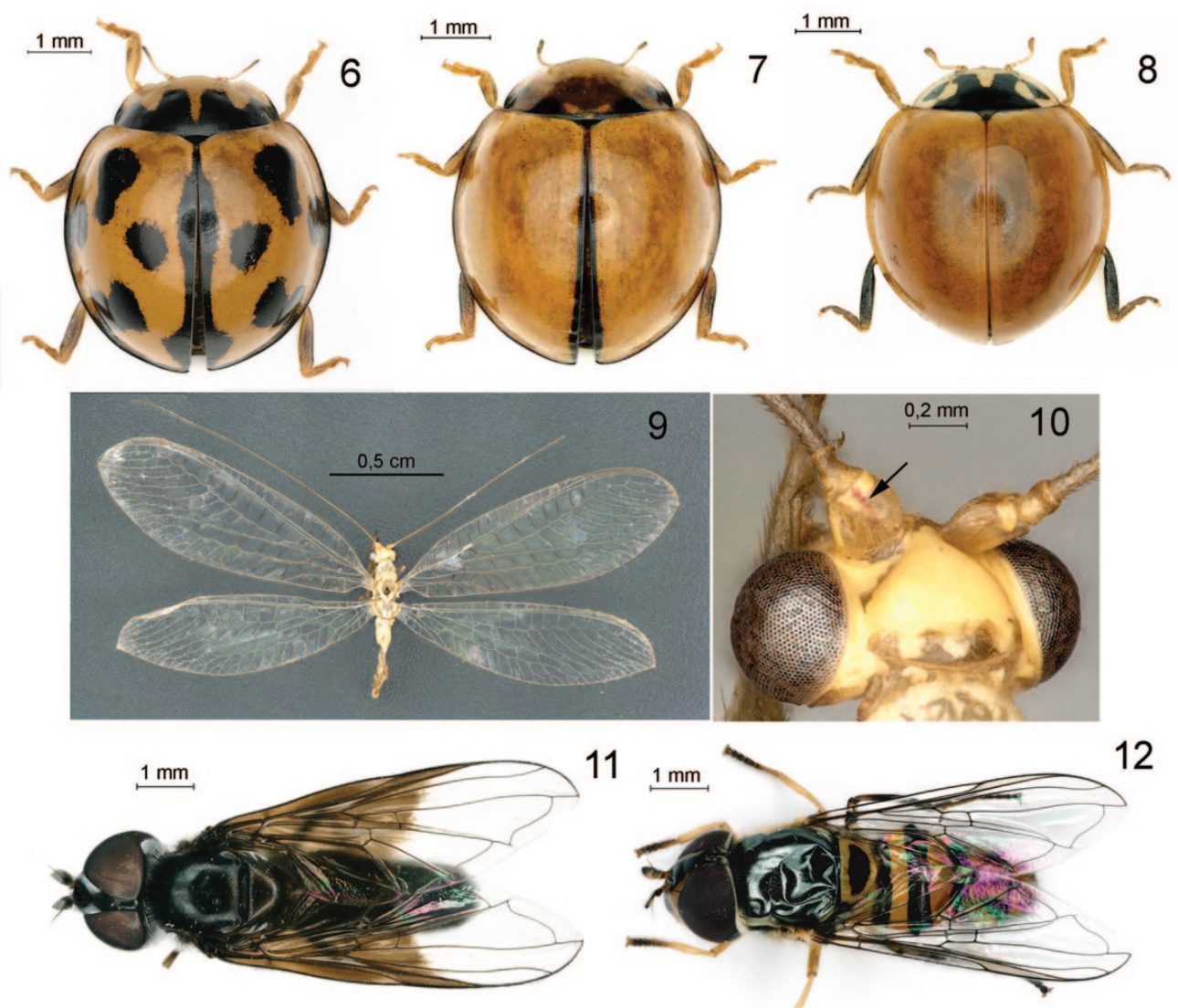

Fig. 6-12. - Prédateurs de Sarucallis kahawaluokalani (Kirkaldy) en Guadeloupe. - 6, Coelophora inaequalis (Coccinellidae). - 7, Cycloneda delauneyi (Coccinellidae). - 8, Cycloneda sanguinea (Coccinellidae). - 9-10, Ceraeochrysa cincta (Chrysopidae) : 9, habitus de l'adulte; 10, tête en vue dorsale (la flèche indique la marque longitudinale sur le scape). - 11, Ocyptamus dimidiatus (Syrphidae). - 12, Allograpta limbata (Syrphidae).

parasitoïdes de $S$. kahawaluokalani dans plusieurs pays, Cameroun, Chine, États-Unis, Jamaïque, Malaisie, Mexique, Philippines, Porto Rico, Thaïlande. Pour expliquer cette absence de parasitisme, ces auteurs avancent trois hypothèses : 1) des parasitoïdes existent mais n'ont pas encore été trouvés, 2) le puceron peut se défendre par encapsulation ou production de toxines, 3) aucun Hyménoptère ne parasite le puceron. Jusqu'à présent aucune de ces hypothèses n'a été vérifiée.

Répartition géographique. - Sarucallis kahawaluokalani est largement répandu dans le monde sur sa principale plante-hôte Lagerstroemia indica. Originaire du Sud-Est asiatique, il a été décrit à partir de spécimens collectés à Hawaii (KIRKALDY, 1907). Outre sa très large répartition sur le continent asiatique, il a également été signalé de la plupart des pays d'Amérique du Sud et centrale, des États-Unis d'Amérique, des Caraïbes, de quelques pays d'Europe (Allemagne, Bulgarie, Croatie, Espagne, France, Grèce, Italie) et du Cameroun (Kondo \& Simbaqueba Cortés, 2014 : Ochaeta, 2017 : Pintar et al., 2015 : Villalobos Muller et al., 2010). En France, sa présence a été signalée pour la première fois par LECLANT \& RENOUST (1986) à partir de captures réalisées sur Lagerstroemia indica au cours des étés 1984 et 1985, à Montpellier et sur le littoral languedocien. 
RemerCiEMENTS. - Nous remercions très vivement nos collègues Eddy Dumbardon-Martial (FREDON-Martinique) pour l'identification des deux espèces de Syrphidae et Michel Martinez pour le prêt de spécimens d'Ocyptamus dimidiatus.

\section{Auteurs CITÉS}

Blackman R.L. \& Eastop V.F., 1994. - Aphids on the world's trees. An identification and information Guide. CAB International in association with The Natural History Museum, London : 986 p.

Étienne J., 2005. - Les Pucerons de Guadeloupe, des Grandes et Petites Antilles (Hemiptera, Aphididae). Bulletin de la Société entomologique de France, 110 (4/5) : 455-462.

Étienne J. \& Champoiseau P., 2011. - Signalement de deux Pucerons nouveaux pour la Guadeloupe (Hemiptera, Aphididae). Bulletin de la Société entomologique de France, 116 (3) : 327-328.

Favret C., 2018. - Aphid Species File. Version 5.0/5.0. http://Aphid.SpeciesFile.org [accédé le 9.X.2018].

Kirkaldy G. W., 1907. - On some peregrine Aphidae in Oahu (Hem.). Proceedings of the Hawaiian Entomological Society, 1 : 99-102.

Kondo T. \& Simbaqueba Cortés R., 2014. - Sarucallis kahawaluokalani (Kirkaldy) (Hemiptera: Aphididae), a new invasive aphid on San Andres island and mainland Colombia, with notes on other adventives species. Insecta Mundi, 0362 : 1-10.

Leclant F. \& Renoust M., 1986. - Le puceron du Lagerstroemia nouveau ravageur pour notre flore. Phytoma, Défense des Cultures, $375:$ 49-50.

Mizell R. F. III, Bennett F. D. \& Reed D. K., 2002. - Unsuccessful search for parasites of the crapemyrtle aphid, Tinocallis kahawaluokalani (Homoptera: Aphididae). Florida Entomologist, 85 : 521-523.

Mizell R. F. III \& Knox G. W., 1993. - Susceptibility of crapemyrtle Lagerstroemia indica L., to the crapemyrtle aphid (Homoptera: Aphididae) in North Florida. Journal of Entomological Science, 28 : 1-7.

Ochaeta J. F. G., 2017. - Primer registro de Sarucallis kahawaluokalani (Kirkaldy, 1907) (Hemiptera: Aphididae) en Lagerstroemia indica (Lythraceae) en Guatemala. Insecta Mundi, 599 : 1-3.

Pintar M., Masten Milek T., Šimala M. \& Seljak G., 2015. - First records of crape myrtle aphid (Sarucallis kahawaluokalani [Kirkaldy, 1907]) and tulip-tree aphid (Illinoia liriodendra [Monell, 1879]) in Croatia. Abstract volume of $12^{\text {th }}$ Slovenian Conference on Plant protection with international participation, Ptuj, Slovenia 3-4 March 2015 : 323-327.

Rojo S., Gilbert F., Marcos-García M. A., Nieto J. M. \& Mier Durante M. P., 2003. - A world review of predatory hoverflies (Diptera, Syrphidae: Syrphinae) and their prey. CIBIO Ediciones, Alicante : 319 p.

Villalobos Muller W., Pérez Hidalgo N., Mier Durante P. \& Nieto Nafría J. M., 2010. - Aphididae (Hemiptera: Sternorrhyncha) from Costa Rica, with new records for Central America. Boletín de la Asociación Española de Entomología, 34 : 145-182.

Zamora Mej́́as D., 2009. - Asociación de áfidos (Aphididae) en Costa Rica, su diversidad e interacciones tróficas con especies parasitoides (Braconidae: Aphidiidae) e hiperparasitoides (Hymenoptera). Tesis, Universidad de Costa Rica, Facultad de Ciencias, Escuela de Biología : 97 p. 